\title{
The expression of SMN1, MART3, GLE1 and FUS genes in spinal muscular atrophy
}

\author{
Aziza Alrafiah ${ }^{1,2}$, Maimonah Alghanmi, \\ Sufana Almashhadi ${ }^{3,4}$, Aqeel Aqeel ${ }^{1}$, Adel Awaji ${ }^{1}$
}

${ }^{1}$ Department of Laboratory Technology, Faculty of Applied Medical Sciences, King Abdulaziz University, Jeddah, Saudi Arabia

${ }^{2}$ Neuroscience Research Unit, Faculty of Medicine, King Abdulaziz University, Jeddah, Saudi Arabia

${ }^{3}$ National Neuroscience Institute, King Fahad Medical City, Riyadh, Saudi Arabia

${ }^{4}$ Institute for Brain Research, Massachusetts Institute of Technology, Cambridge, USA

\begin{abstract}
Introduction. Spinal muscular atrophy (SMA) is one of the most common genetic causes of death in infants due to a mutation of the motor neuron 1 (SMN1) gene. The SMN1 gene encodes for the multifunctional SMN protein. SMN has been shown to be implicated in pre-mRNA splicing, mRNA transport and translational control. Also other mRNA processing proteins, such as GLE1, Marten (MART3) and Fused in Sarcoma (FUS), have been linked to neurodegenerative diseases. The aim of the study was to determine the expression of $S M N$, GLE1, MART3 and FUS genes in cell lines of the fibroblasts derived from SMA patients and normal controls. Material and methods. Total RNA was extracted from purchased fibroblasts acquired from three SMA type I patients and fibroblasts of three age-matched healthy controls. The RNA was then subjected to qPCR analysis using primers specific for the GLE1, MART3, FUS and SMN1 genes vs. GAPDH as internal control gene.

Results. SMN1 mRNA levels were at least $\times 10$ lower in fibroblasts of SMA patients compared to controls. Gle1 and MART3 gene expression was $\times 2$ downregulated whereas FUS mRNA levels appeared to be $\times 3$ upregulated in SMA cells when compared to controls. We found a high correlation between FUS gene expression level to the SMN1 at gene expression level of fibroblast cell lines of SMA type I patients $(\mathrm{r}=0.994, \mathrm{p}<0.0001)$.

Conclusions. Our preliminary data show an intriguing expression profile of Gle1, MART3 and FUS genes in SMA, and suggest a critical role of FUS protein in the SMA pathogenesis. (Folia Histochemica et Cytobiologica 2018, Vol. 56, No. 4, 215-221)
\end{abstract}

Key words: Spinal muscular atrophy; fibroblasts; GLE1; SMN1; FUS; MART3; qPCR

\section{Introduction}

Spinal muscular atrophy (SMA) is a neurodegenerative disease primarily affecting the lower motor neurons, leading to weakness, atrophy and eventually paralysis. Patients suffering from SMA typically die before the age of two years [1]. To date, evidence

Correspondence address: A. Alrafiah

PO Box 80200, Jeddah 21589, Saudi Arabia

tel.: + $966(01) 26401000$ Ext. 23495

fax: +966 (01) 26401000 Ext. 21686

e-mail: aalrafiah@kau.edu.sa shows that mutations of the motor neuron 1 (SMN1) gene is the main cause of pathology. The SMN1 gene encodes for a multifunctional SMN protein [1].The earliest reported and best characterized role of SMN1 is in pre-mRNA splicing, but it has also been implicated in mRNA transport and translational control [1].

The clinical severity of SMA can vary widely, ranging from death in infancy to disability in adulthood. The disease has been classified into: Type I, Type II, Type III and Type IV, depending on the clinical manifestations and age at onset of disease [2]. SMA type 1 starts to affect neonates up to the age of six months. It has been reported that SMN1 gene that encrypts the SMN protein and plays a vital role in 
transcriptional regulation, telomerase regeneration and cellular trafficking, is either deleted or missing [2]. Although the genetic basis of SMA is well understood, the underlying mechanism of pathology of the remains unclear $[2,3]$. Moreover, it is not yet fully understood how the lack of SMN leads to SMA, and why is it affecting solely the spinal motor neurons. There is an urgent need to further investigate the mechanism and molecular component of the disease, which could open new therapeutic avenues.

Mounting research evidence suggests that SMN performs a significant function both within pre-mRNA splicing and in the axon's function of trafficking mRNA-binding proteins and their target mRNAs $[3,4]$. The challenge currently facing researchers is to understand why a defect in the spliceosome machinery used by all cells should selectively affect the motor neurons $[3,4]$.

Research has shown several genetic predispositions common between neurodegenerative diseases. For example, mutations to chromosome 9 hexanucleotide repeat72 (C9ORF72), transactive response DNA binding protein $(T A R D B P)$ and Charged Multivesicular Body Protein 2B (CHMP2B) have been implicated in both frontotemporal lobar degeneration (FTLD) and amyotrophic lateral sclerosis (ALS) [5]. Mutations to the ataxin-2 gene (ATXN2) have been reported in ALS and spinocerebellar ataxia type 2 (SCA2) [6]. Furthermore, many neurodegenerative disease-related genetic risk factors have roles in RNA processing, such as FUS, TARDBP, C9orf72, angiogenin (ANG), ataxin-2 (ATXN2), matrin 3 (MART3), huntingtin, and SMN1 [6-11]. This underscores the importance of efficient RNA-processing for a healthy nervous system.

In this paper, we selected three genes: GLE1 (RNA export mediator) [12], FUS (Fused in Sarcoma) [13] and MART3 (Mammalian retrotransposon transcript) [14], which are known to be involved in other neurodegenerative diseases, with the aim of examining them for differential expression between normal human fibroblasts and primary fibroblast cultures derived from the skin of SMA patients. GLE1 encodes for a protein that is involved in RNA export, and mutations to this gene have been associated with lethal congenital contracture syndrome 1 (LCCS1) $[11,15,16]$. FUS mutations have been associated with few juvenile ALS cases [11]. MART3, meanwhile, is a DNA and RNA binding protein with diverse RNA processing functions. It has been reported to interact with TDP-43 [5]. Mutations in the MART3 gene have been linked to some ALS cases [7].

To date, clinical trials have very limited success in reporting an effective treatment of neurodegenerative disorders. However, a lot of effort has been made in trying to understand the molecular mechanism of these diseases in order to target different therapeutic approaches that are based on the evidence that SMN1 expression levels correlate with SMA severity [4]. Therapeutic development is focused mainly on increasing SMN protein levels using gene therapy based on SMN1 replacement by viral vectors [17]. Other therapeutic trials focus on cell replacement and neuroprotection $[17,18]$.

This case-control study was undertaken to investigate the association between the expression of the SMN1 gene which controls the fate of mRNA, and other mRNA processing genes such as GLE1, MART3 and FUS that have been linked to other motor neuron diseases.

\section{Material and methods}

Cell lines. Human fibroblasts of three healthy subjects (Cat. No: GM05565, GM00498 \& AG08498) and three age-matched type I SMA patients (3 years old, male) were purchased from the Coriell Institute for Medical Research (Cat. No GM03813, GM03814 \& GM03815). Cells were cultured in tissue culture flasks T75 in minimal essential medium (MEM) (M2279, Sigma-Aldrich, St. Louis, MO, USA) containing $10 \%$ fetal bovine serum (FBS) (FB1001, Biosera, Kansas City, KS, USA), $1 \%$ penicillin-streptomycin (17-602E, Lonza, Basel, Switzerland), 1\% MEM Eagle Vitamin Mixture (13-607C, Lonza), 1\% sodium pyruvate (Sigma) and $0.1 \%$ uridine $(50 \mathrm{mg} / \mathrm{mL}$, Sigma).

\section{RNA extraction and quantitative polymerase chain reaction} (qPCR). Tissue samples were harvested in $1 \mathrm{ml} \mathrm{GIBCO} \mathrm{BRL}$ TRIZOL (Cat. No. 15596) per 50 to $100 \mathrm{mg}$ tissue, using a glass-TEFLON homogenizer (Thomas Scientific, Swedesboro, NJ, USA). After 5 min incubation at room temperature (RT) of the homogenized samples chloroform was included with a portion of $0.2 \mathrm{ml}$ for every $1 \mathrm{ml}$ of TRIZOL Reagent. The mixture was shocked actively followed by centrifugation for $15 \mathrm{~min}$ at $4^{\circ} \mathrm{C}$ at $12,000 \mathrm{rpm}$.

RNA was collected from the top aqueous stage followed by mixing with $0.5 \mathrm{ml}$ isopropanol in new Eppendorf flask and incubated for $10 \mathrm{~min}$ at RT, then re-centrifuged for $20 \mathrm{~min}$ at $13,300 \mathrm{rpm}$ at $4^{\circ} \mathrm{C}$.

The RNA pellet was washed with $75 \%$ alcohol and dried at RT then thawed in diethyl pyrocarbonate (DEPC)-treated water. The RNA was quantified using the NanoDrop spectrophotometer ND2000 (Labtech, East Sussex, UK). After that following the standard Quick-Start supplied protocol, RNA $700 \mathrm{ng} / \mathrm{microL}$ was reversibly transcribed to complimentary DNA (cDNA) using the QuantiTect Reverse Transcription Kit (Cat No./ID: 205310, Qiagen, Hilden, Germany), Real-time PCR was performed in a MX3000P 


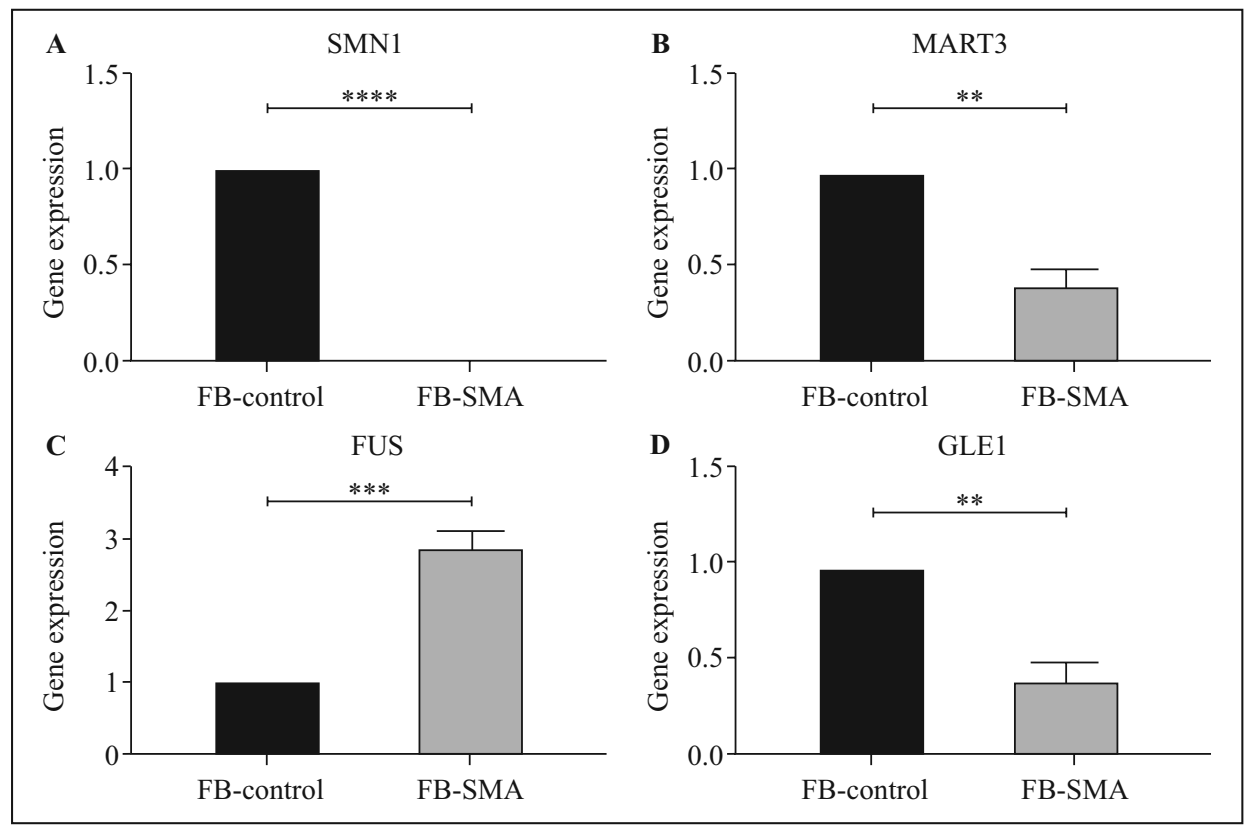

Figure 1. The expression of the studied genes was determined by qPCR in SMA fibroblasts of spinal muscular atrophy patients (FB-SMN) and control fibroblasts (FB-Control). A. SMN gene expression. The bar chart shows that the SMN1 expression (Y axis) was absent in SMA fibroblasts (FB-SMA) and present in the control samples (FB-Control); ***p < 0.0001 (paired $\mathrm{t}$-test, $\mathrm{n}=3$ ). Error bars represent the standard error of the mean. B. MART3 gene expression. It was down-regulated in SMA fibroblasts in relation to control fibroblasts; ${ }^{* *} \mathrm{p}<0.005$ (paired t-test, $\mathrm{n}=3$ ). C. FUS gene expression. It was up-regulated in SMA fibroblasts $(* * * \mathrm{p}<0.001$ (paired t-test, $\mathrm{n}=3$ ). D. GLE1 gene expression. It was down regulated in SMA fibroblasts $(* * \mathrm{p}<0.005$ two-way ANOVA, $\mathrm{n}=3)$.

RT-PCR System (Stratagene, San Diego, CA, USA) to evaluate the expression levels of SMN1, MART3, GLE1 and $F U S$, with the levels being normalized against glyceraldehyde phosphate dehydrogenase $(G A P D H)(\mathrm{n}=3)$.

The Primer sequences used for each gene were as follows:

SMN1 cDNA was amplified using the primer pair: SMN1 human F 5'-GAGGAGCAAAATCTGTCC-3', SMN1 human R 5'-TTTCCAGGAGACCTGGAG-3'; GLE11 human F 5'CTTTGACAAGATCCACAG, GLE1 human R5' CACCATGAAGCAGCATTC;

MART3 human 5'GATGACTTGAAAGTAGGG, MART3 human R5'GTGTGACACCAAGATAAG;

FUS human 5'AAACAAGAAAACGGGACAGC, $F U S$ human R5'GGTCTCATTTGCTACTCGCC.

The primer pair b-GAPDH mRNA, F- 5' GGAAGCTCACTGGCATGGC3', and the R-5' TAGACGGCAGGTCAGGTCCA 3' was used as the housekeeping transcript.

All primers were added to a final concentration of $100 \mathrm{nM}$ with approximately $12.5 \mathrm{ng}$ of cDNA per well. Real-time polymerase chain reaction (RT-PCR) reaction was performed in the volume of $13 \mu$ l with a Brilliant II SYBR Green QPCR master mix (Stratagene) for 40 cycles of 2-step qPCR protocol. Using the Delta-Delta-Ct (DDCt) method the relative expression was calculated by ABI PRISM 7700
Sequence Detection System protocol (Applied Biosystems, Foster City, CA, USA). Each sample was run in triplicate.

Statistical analysis. All data are presented as mean \pm SD. Each gene was analyzed using GraphPad Prism v. 7.02 software (Graphpad, San Diego, CA, USA). Differences were analyzed by the paired t-test to contrast every single SMA fibroblast line with the control fibroblast cell line and by one-way ANOVA with $\mathrm{p}$ less than 0.05 regarded as statistically significant.

\section{Results}

All experiments were performed in triplicate $(n=3)$.

\section{SMN1 gene expression in fibroblasts \\ of SMA patients}

SMN1 gene expression levels were at least $\times 10$ lower and were even barely detected in the SMA fibroblasts compared to control fibroblasts samples (Fig. 1A, $\mathrm{p}<0.0001)$.

\section{MART3 gene expression in SMA fibroblasts}

$M A T R 3$ expression levels in the SMA fibroblasts were found to be $\times 2$ lower as compared to the control fibroblasts samples (Fig. 1B, p < 0.005). 


\section{FUS gene expression in SMA fibroblasts}

Interestingly, FUS expression underwent a $\times 3$ upregulation in the SMA fibroblasts compared to control fibroblasts samples (Fig. 1C, $\mathrm{p}<0.0001$ ).

\section{GLE1 gene expression in SMA fibroblasts}

GLE1 expression was down regulated in SMA fibroblasts, approximately $\times 2$ of its expression in control fibroblasts (Fig. 1D, $\mathrm{p}<0.005$ ).

\section{Associations between expression of FUS and MART3 genes and SMN1 gene expression}

Multiple regression analysis was used to test if the $S M N 1$ gene expression at mRNA level significantly predicted gene level expression of GLE1, MART3 and FUS proteins.

Single linear regression test was performed to assess correlation between FUS and SMN1 genes and indicated that there was very high correlation coefficient R2 of 0.994, $p<0.0001$ (Fig. 2A).

Single linear regression test was performed to assess correlation between MART3 and SMN1 genes expression. There was a moderate positive correlation $\mathrm{R} 2$ of $0.592, \mathrm{p}=0.005$ (Fig. 2B).

Single linear regression test was performed to assess correlation between GLE1 and SMN1 genes expression. We found that there was a weak correlation $\mathrm{R} 2$ of $0.354, \mathrm{p}=0.01$.

\section{Discussion}

The mechanism by which SMN1 gene alteration leads to SMA pathological state has yet not been completely explained. In the current research, we aimed to contribute to the current body of knowledge of SMA and increase our understanding of the pathogenesis of the disease. This study reports on the differential expression of four genes involved in mRNA processing, SMN1, MART3, GLE1 and FUS genes, in SMA patients fibroblast cell line compared to the age-matched control cell line. Our results showed a correlation between genetic risk factors of neurodegenerative diseases: FUS, MATR3 and GLE1 which are nuclear proteins that are involved in RNA processing. FUS protein binds to DNA and regulates transcription $[19,20]$. MATR3 has several functions in post-transcriptional RNA processing [15]. Interaction between FUS and MATR3 has been reported recently [21], whereas GLE1 is involved in mRNA export and translation $[12,22]$.

Considering the functions of the four genes of interest in this study, i.e. FUS involved in transcription, SMN and MATR3 involved in post-transcriptional RNA processing and GLE1 involved in mRNA ex-

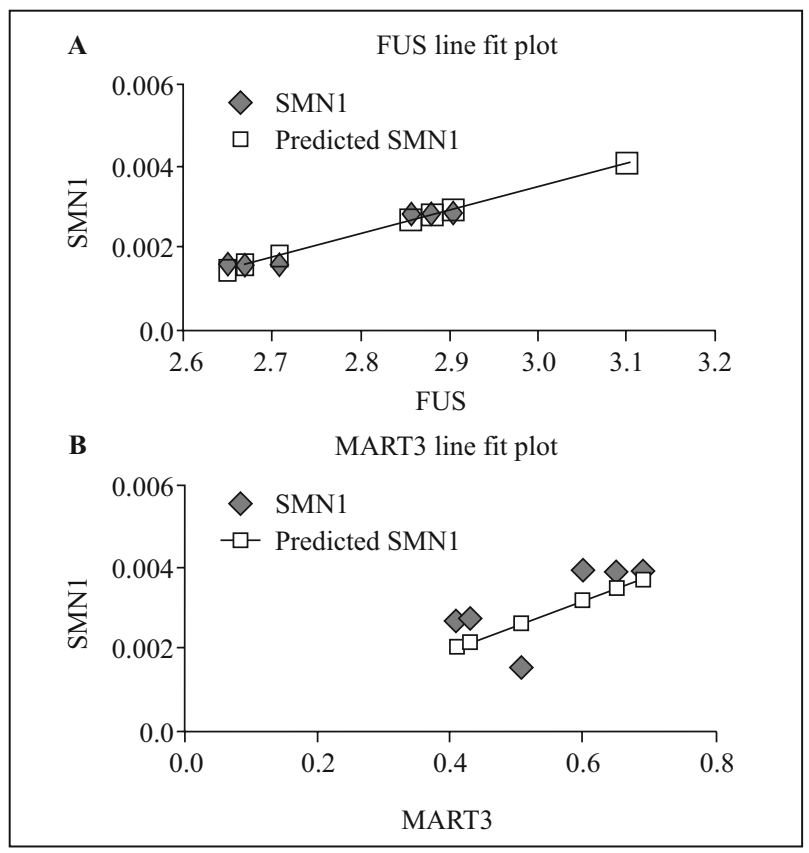

Figure 2. Prediction of the expression of FUS and MART3 genes on SMN1 gene and protein expression. Multiple regression analysis was used to test if the SMN1 gene expression levels significantly predicted expression of FUS (A) and MART3 (B) proteins. A. Significant very strong positive correlation of SMN1 and FUS mRNAs is shown ( $p=0.00001, \mathrm{R} 2=0.986)$. The diamond-shaped symbols represent SMN1 mRNA and square symbols predicted SMN1 protein levels. B. Moderate correlation between SMN1 and MART3 mRNAs was found $(\mathrm{p}=0.005, \mathrm{R} 2=0.592)$. The diamond-shaped symbols represent SMN1 mRNA and square symbols predicted SMN1 protein levels $\mathrm{N}=3$.

port, our results show that FUS gene responds to the absence of SMN level by increased expression of its mRNA while genes encoding MATR3 and GLE1 decrease their expression what is suggestive of the interaction between these proteins.

Particularly, we documented differential expression of these genes in the SMA patient-derived fibroblast cell line with dramatic downregulation of the SMN1 gene and significant downregulation of the GLE1 and MART3 gene expression. On the contrary, the FUS gene expression was found to be upregulated by three-fold in the SMA patient derived fibroblasts. The statistical analysis indicated that both MATR3 and GLE1 genes expression levels in fibroblast cell lines of type I SMA patient are not significant predictors of SMN1 gene expression level. However, FUS gene expression level in fibroblast cell lines of type I SMA patient was found to be a very significant predictor of SMN1 gene level expression.

The various levels of aberrant gene expression are correlated and the characteristic of the dysregulation 
of mRNA processing proteins in various disease states [23]. Our study has confirmed dysregulated expression of the studied genes in SMA, which suggests that these genes may play a role in the disease pathogenesis. Ultimately, these genes can serve as potential biomarkers and therapeutic targets to aid in disease diagnosis and treatment at the prenatal or new-born stage.

Interestingly, the marked downregulation of SMN1 expression in the context of SMA in this study is in concert with the findings of the study by Lefebvre $e t$ al. (1995) [4] who first reported on SMN1 deficiency in SMA. This pioneer SMA study identified and characterized a $20 \mathrm{~kb}$ gene, now known to be a gene coding for SMN protein [4].

The interaction between FUS and SMN proteins in HeLa cells and different mouse tissues including the brain and spinal cord has been reported previously [24]. It was reported that ALS-linked FUS mutations altered the interaction between FUS and SMN [25]. The findings in our study showed that FUS is overexpressed in SMA fibroblasts, in accordance with the findings Mirra et al. [25]. This suggests that FUS has a molecular pathogenicity similar to SMN1 in causing SMA [25].

MART3 gene, on the other hand, has never been studied in the context of SMA patients, but it was proposed by Mirra et al. [25] to be implicated in SMA. The detected halved expression of MART3 in SMA fibroblasts in our experiment suggests that it has a role to play in SMA pathogenesis. The results of our study clearly show that the three genes are differentially expressed in the context of SMA. Thus, it is possible that these genes can be developed as biomarkers for SMA disease diagnosis and eventually, as targets of drug treatments against SMA.

In this context it is worth to mention that Ogino et al. suggested that genetic testing is an essential component of the proper management of SMA symptoms [3]. Thus, if we combine our findings with the current knowledge on SMA genetic dysregulation and include analysis of FUS and MART3 gene expression in the battery of biomarker tools used for SMA diagnosis, along with $S M N 1$, increased SMA detection accuracy could be achieved. Moreover, if the roles of these mRNA processing proteins in SMA pathogenicity have been well defined and elucidated, the dependability factor in predicting SMA using these biomarkers will be much improved. Eventually, these findings and their potential applications in terms of diagnostics and therapy could help to manage and minimize the severity of the disease.

As suggested by Johnson et al. [9] and Gama-Carvalho et al. [26], SMA and ALS have multiple links and shared abnormalities. These abnormalities are mostly based on the genetic changes in and dysregulation of their RNA processing proteins encoded by such genes as $C 9$ orf72, MART3 and FUS. Therefore, a wider investigation on the correlation of the ALS-associated genes and other neurodegenerative diseases-related genes is necessary.

The current study revealed by applying quantitative real-time PCR that the dysregulated expression of SMA, MART3 and FUS was found within SMA patient-derived fibroblast cell-lines as compared to fibroblast of a healthy control. A similar approach can be adapted to study the putative proteins implicated in different motor neuron disorders such as ALS. Despite their stark similarities in neurodegeneration symptoms, different motor neuron diseases are defined through different causative genetic lesion/s [26]. Thus, differences in the gene and protein expression profiles are expected to be obtained in different disease contexts.

While the exact disease pathogenesis for SMA due to mRNA dysregulation has still not been fully understood, dysfunctional gene expression can cause a broad range of disorders. In order to maintain the gene expression in specific cell states in humans it is controlled by thousands of cofactors, chromatin regulators and transcription factors. Moreover, transcription dysfunction phenomena may hold clues as to the severity and onset of neuronal muscular disorders although this has not yet been conclusively determined. Nonetheless, the findings of the current study provide additional knowledge that can aid further investigations into the disease pathogenesis of SMA, and more importantly, a basis for clinical detection in individuals suspected to be at risk.

As presented by Marketou et al., GLE1 could be a promising gene therapy target to provide neuroprotection in infants with SMA [18]. Similarly, MART3 and FUS must be studied further to obtain more information and ensure the credibility of the results before they can be used for in vivo clinical tests.

While numerous studies have attempted to provide a detailed background for understanding SMA pathogenicity $[7,26]$, our study has demonstrated an intriguing association between the differential gene expression of SMN1, MART3, and FUS in SMA that may contribute to the mechanisms of SMA pathogenicity.

\section{Conclusions}

Our results emphasize the importance of hypothesis that changes of the RNA processing proteins play a key role in SMA pathomechanisms. Further studies 
would show if these proteins can be used as monitoring biomarkers early at the pregnancy stage to diagnose SMA and thus increase the survival rate if the treatment is available.

\section{Acknowledgements}

We thank Deanship of Scientific Research for funding Neuroscience Research Unit at King Abdulaziz University for providing facilities for the current project. The authors thank Dr. Ebtisam Alofi and Dr. Ahmed Mirza for their assistance with statistical analysis and Dr. Kalthom Alsaqaf for her support to the Msc Student Aqeel Aqeel.

This Project was funded by the Deanship of Scientific Research (DSR), at King Abdulaziz University, Jeddah, under grant no. (G-587-290-38). The authors, therefore, acknowledge with thanks DSR for technical and financial support.

\section{Declarations}

Ethics approval and consent to participate. Coriell Institute (Camden, NJ 08103, USA) has obtained the original fibroblast cell lines for Medical Research. Regarding policy of informed consent form, the Coriell Institute for Medical Research, has obtained a Certificate of Confidentiality from the National Institutes of Health to help ensure patient's privacy.

\section{Competing interests}

The authors declare that they have no competing interests.

\section{Authors' contributions}

A. Alrafiah is the first and corresponding author. The work was distributed equally between authors. All authors read and approved the final manuscript.

\section{References}

1. Hamilton G, Gillingwater TH. Spinal muscular atrophy: going beyond the motor neuron. Trends Mol Med. 2013; 19(1): 40-50, doi: 10.1016/j.molmed.2012.11.002, indexed in Pubmed: 23228902.

2. Liu H, Shafey D, Moores JN, et al. Neurodevelopmental consequences of Smn depletion in a mouse model of spinal muscular atrophy. J Neurosci Res. 2010; 88(1): 111-122, doi: 10.1002/jnr.22189, indexed in Pubmed: 19642194.

3. Ogino S, Wilson RB. Genetic testing and risk assessment for spinal muscular atrophy (SMA). Hum Genet. 2002; 111(6): 477-500, doi: 10.1007/s00439-002-0828-x, indexed in Pubmed: 12436240 .

4. Lefebvre S, Bürglen L, Reboullet S, et al. Identification and characterization of a spinal muscular atrophy-determining gene. Cell. 1995; 80(1): 155-165, indexed in Pubmed: 7813012.
5. Liscic RM, Grinberg LT, Zidar J, et al. ALS and FTLD: two faces of TDP-43 proteinopathy. Eur J Neurol. 2008; 15(8): 772-780, doi: 10.1111/j.1468-1331.2008.02195.x, indexed in Pubmed: 18684309.

6. Elden AC, Kim HJ, Hart MP, et al. Ataxin-2 intermediate-length polyglutamine expansions are associated with increased risk for ALS. Nature. 2010; 466(7310): 1069-1075, doi: 10.1038/nature09320, indexed in Pubmed: 20740007.

7. Boehringer A, Garcia-Mansfield K, Singh G, et al. ALS Associated Mutations in Matrin 3 Alter Protein-Protein Interactions and Impede mRNA Nuclear Export. Sci Rep. 2017; 7(1): 14529, doi: 10.1038/s41598-017-14924-6, indexed in Pubmed: 29109432.

8. Guerreiro R, Brás J, Hardy J. SnapShot: Genetics of ALS and FTD. Cell. 2015; 160(4): 798-798.e1, doi: 10.1016/j. cell.2015.01.052, indexed in Pubmed: 25679767.

9. Johnson JO, Pioro EP, Boehringer A, et al. ITALSGEN. Mutations in the Matrin 3 gene cause familial amyotrophic lateral sclerosis. Nat Neurosci. 2014; 17(5): 664-666, doi: 10.1038/ nn.3688, indexed in Pubmed: 24686783.

10. Liu EY, Cali CP, Lee EB. RNA metabolism in neurodegenerative disease. Dis Model Mech. 2017; 10(5): 509-518, doi: 10.1242/dmm.028613, indexed in Pubmed: 28468937.

11. Bäumer D, Hilton D, Paine SML, et al. Juvenile ALS with basophilic inclusions is a FUS proteinopathy with FUS mutations. Neurology. 2010; 75(7): 611-618, doi: 10.1212/WNL. 0b013e3181ed9cde, indexed in Pubmed: 20668261.

12. Murphy R, Wente SR. An RNA-export mediator with an essential nuclear export signal. Nature. 1996; 383(6598): 357-360, doi: 10.1038/383357a0, indexed in Pubmed: 8848052.

13. Mirra A, Rossi S, Scaricamazza S, et al. Publisher Correction: Functional interaction between FUS and SMN underlies SMA-like splicing changes in wild-type hFUS mice. Sci Rep. 2018; 8(1): 7005, doi: 10.1038/s41598-018-25176-3, indexed in Pubmed: 29712963.

14. Belgrader, P., R. Dey, and R. Berezney, Molecular cloning of matrin 3. A 125-kilodalton protein of the nuclear matrix contains an extensive acidic domain. J Biol Chem, 1991. 266(15): p. : 9893-9.

15. Salton M, Elkon R, Borodina T, et al. Matrin 3 binds and stabilizes mRNA. PLoS One. 2011; 6(8): e23882, doi: 10.1371/ journal.pone.0023882, indexed in Pubmed: 21858232.

16. Seytanoglu A, Alsomali NI, Valori CF, et al. Deficiency in the mRNA export mediator Gle1 impairs Schwann cell development in the zebrafish embryo. Neuroscience. 2016; 322: 287-297, doi: 10.1016/j.neuroscience.2016.02.039, indexed in Pubmed: 26921650.

17. Foust KD, Wang X, McGovern VL, et al. Rescue of the spinal muscular atrophy phenotype in a mouse model by early postnatal delivery of SMN. Nat Biotechnol. 2010; 28(3): 271-274, doi: 10.1038/nbt.1610, indexed in Pubmed: 20190738.

18. Lukashchuk V, Lewis KE, Coldicott I, et al. AAV9-mediated central nervous system-targeted gene delivery via cisterna magna route in mice. Mol Ther Methods Clin Dev. 2016; 3: 15055, doi: 10.1038/mtm.2015.55, indexed in Pubmed: 26942208

19. Schwartz JC, Ebmeier CC, Podell ER, et al. FUS binds the CTD of RNA polymerase II and regulates its phosphorylation at Ser2. Genes \& Development. 2012; 26(24): 2690-2695, doi: 10.1101/gad.204602.112.

20. $\mathrm{Yu} \mathrm{Y,} \mathrm{Reed} \mathrm{R.} \mathrm{FUS} \mathrm{functions} \mathrm{in} \mathrm{coupling} \mathrm{transcription} \mathrm{to}$ splicing by mediating an interaction between RNAP II and U1 snRNP. Proc Natl Acad Sci U S A. 2015; 112(28): 8608-8613, doi: 10.1073/pnas.1506282112, indexed in Pubmed: 26124092. 
21. Yamaguchi A, Takanashi K. FUS interacts with nuclear matrix-associated protein SAFB1 as well as Matrin3 to regulate splicing and ligand-mediated transcription. Sci Rep. 2016; 6: 35195, doi: 10.1038/srep35195, indexed in Pubmed: 27731383 .

22. Bolger TA, Folkmann AW, Tran EJ, et al. The mRNA export factor Gle1 and inositol hexakisphosphate regulate distinct stages of translation. Cell. 2008; 134(4): 624-633, doi: 10.1016/j.cell.2008.06.027, indexed in Pubmed: 18724935.

23. Zeng Y, Wang G, Yang E, et al. Aberrant gene expression in humans. PLoS Genet. 2015; 11(1): e1004942, doi: 10.1371/ /journal.pgen.1004942, indexed in Pubmed: 25617623.
24. Sun S, Ling SC, Qiu J, et al. ALS-causative mutations in FUS/ TLS confer gain and loss of function by altered association with SMN and U1-snRNP. Nat Commun. 2015; 6: 6171, doi: 10.1038/ncomms7171, indexed in Pubmed: 25625564.

25. Mirra A, Rossi S, Scaricamazza S, et al. Functional interaction between FUS and SMN underlies SMA-like splicing changes in wild-type hFUS mice. Sci Rep. 2017; 7(1): 2033, doi: 10.1038/s41598-017-02195-0, indexed in Pubmed: 28515487.

26. Gama-Carvalho M, L Garcia-Vaquero M, R Pinto F, et al. Linking amyotrophic lateral sclerosis and spinal muscular atrophy through RNA-transcriptome homeostasis: a genomics perspective. J Neurochem. 2017; 141(1): 12-30, doi: 10.1111/ /jnc.13945, indexed in Pubmed: 28054357.

Submitted: 14 July, 2018

Accepted after reviews: 29 October, 2018 Available as AoP: 26 November, 2018 